\title{
PERFORMANCE OF UNCORRUPTED PIXEL COUNT DECISION BASED FILTER FOR REMOVAL OF SALT AND PEPPER NOISE FROM DIGITAL IMAGE
}

\author{
Ramkumar $^{1}$, Rajesh $^{2}$, Sharmila $^{3}$ \\ ${ }^{1} P G$ Scholar Dept of ECE, Manakula Vinayagar Institute of Technology, Puducherry, India \\ ${ }^{2}$ Assistant Professor, Dept of ECE, Manakula Vinayagar Institute of Technology, Puducherry, India \\ ${ }^{3} P G$ Scholar Dept of ECE, Manakula Vinayagar Institute of Technology, Puducherry, India
}

\begin{abstract}
Uncorrupted pixel count Decision Based Filter (UPCDBF) algorithm employed in the process of removal of noise densities and restoration of grey scale image Which are heavily corrupted by salt and pepper noise. The algorithm replaces the corrupted centre pixel by midpoint or median value based upon the noise free pixel count. This algorithm outperforms Standard median filter $(M F)$, Arithmetic median(AMF) and PSMF. The algorithm exhibits High PSNR value.
\end{abstract}

Keywords: Salt and pepper noise, uncorrupted pixel count decision based filter (UPCDBF)

\section{INTRODUCTION}

Image processing field have tremendous growth day by day by advancements in capturing devices and numerous applications. But there are still some bottlenecks on which researchers have their focus. In digital image the factors such as imperfections in imaging sensors, channel transmission errors and faulty memory locations in hardware leads to salt and pepper noise. The corrupted pixels take either maximum or minimum grey level, which affects the information of the image.

\subsection{Image Filtering}

Images are often corrupted by salt and pepper noise due to error in transmission of digital images. In yester years removal of noise is facilitated, by using linear filtering techniques. These linear approaches were very popular because of its mathematical simplicity. The objective of noise removal is to eliminate the salt and pepper noise with minimum deformation caused to the image. The linear filters were not effective in removing non Gaussian noise. The removal of impulse noise leads to blurred and defamed features of the image. Hence nonlinear filters were introduced. The most widely used nonlinear filters are median filters. Median filters would eliminate impulse and preserves edges in the image. For increasing noise densities the standard median filter (SMF) flatters also irrespective of pixel is noisy or not, median is applied to entire image [1].

An adaptive median filter (AMF) removed impulse noise at high noise densities, owing to its increasing window size caused blurring of images [2]. Many switched median filters were proposed to detect and correct only the corrupted pixel [3]-[4]. The flaw of the switched median filters is that it detected and corrected the impulse, but did not take local feature such as edges into account. For the removal of high density impulse noise (DBA) Decision based filters [5] were proposed. Due to the replacement of neighborhood pixel the performance of the image tend to flatter above $60 \%$ by exhibiting "Streaks" in images. Hence the edges of the images are destroyed. To avoid streaking in images at heavy noise conditions Uncorrupted Pixel Count Decision Based Filter is proposed (UPCDBF). Where it functions based on the remaining pixel after removal of corrupted pixels.

\subsection{Noise Model}

Salt and pepper noise with equal noise probability:

If [0 255] denote the dynamic range of y',

i.e., $0<=\mathrm{Sij}<=255$ for all $(\mathrm{i}, \mathrm{j})$,then they are denoted by Saltand-pepper noise

$\mathrm{Y}_{\mathrm{ij}}=0 \quad$ with probability $\mathrm{p}$;

$\mathrm{S}_{\mathrm{ij}} \quad$ with probability $1-\mathrm{p}-\mathrm{q}$;

$255 \quad$ with probability q;

Where $\mathrm{s}=\mathrm{p}+\mathrm{q}$ denotes the salt-and-pepper noise level.

\section{UNCORRUPTED PIXEL COUNT DECISION BASED FILTER (UPCDBF)}

The pixels of processing window are arranged in 1D form if the centre pixel is found to be corrupted pixel. The pixels are processed to 1D using snake like sorting technique. Then after removal of corrupted pixels the algorithm is carried out with count of noise free pixels. 


\section{Snake like improved Shear Sorting}

Over the years sorting algorithm is a basic operation behind all the median filters. All the existing sorting algorithms require more comparators. In this paper a new snake like improved shear sorting algorithm is used for ordering the entire array of processed pixels.

The algorithm of snake like improved shear sorting algorithm is as follows.

Step1: The considered 2D processing window(3X3).

Step2: Sort the 1th and 3rd rows of the 2D array in ascending order and 2 nd row in descending order independently .The sorted sequence is fed to step3.

Step3: Sort the three columns of the 2D array in ascending order . The sorted sequence is fed to step4.

Step4: Repeat step 2 and 3 once again as shown in figure1.d and e.

Step5: Now Sort the upper semi diagonal of the semi sorted 2D array in ascending order.

Step6: Sort the Lower semi diagonal sorted array in ascending order. Resulting array is sorted in a snake like order. The procedure is repeated for the other windows of the image [9].

\subsection{Proposed Algorithm}

The Uncorrupted pixel count decision based filter initially detects impulse and corrects it subsequently. All the pixels of an image lie between the dynamic ranges $[0,255]$. If the processed pixel holds minimum (0) or maximum (255), pixel is considered as noisy and processed by UPCDBF else as not noisy and the pixel is unaltered. The brief illustration of the algorithm is as follows.

Step 1: Choose 2-D window of size $3 \times 3$. The processed pixel in current window is assumed as pxy.

Step 2: Check for the condition $0<\mathrm{pxy}<255$, if the condition is true then pixel is considered as not noisy and left unaltered.

Step 3: If the processed pixel pxy holds 0 or 255 i.e. (pxy=0 or pxy $=255$ ) then pixel pxy is considered as corrupted pixel. Convert 2D array into 1D array. Sort the 1D array which is assumed as Sxy.

Step 4: Initialize two counters, forward counter $(\mathrm{F})$ and reverse counter (L) with 1 and 9 respectively. When a 0 or 255 is encountered inside the window $\mathrm{F}$ is increased by 1 or $\mathrm{L}$ is decremented by 1 respectively. When pixel is noisy there happens to be two possible cases. Check for the number of corrupted pixel inside the current processing window. It is denoted as count.

Case 1) if the Uncorrupted value (9-count) is even number then in current processing window the corrupted pixel is replaced with median of uncorrupted pixels as output.

Case 1) if the Uncorrupted value (9-count) is odd number then in current processing window the corrupted pixel is replaced with mid point value of uncorrupted pixels as output. If entire pixels inside the processing window are 0 or $255 \mathrm{ie})(9$-count $=0$ ) then pixel value is retained considering it as texture.
Step 5: Steps 1 to 4 is repeated until all pixels of the entire image is processed.

\subsection{Insight of UPCDBF}

The processed pixel is checked for low (0) or high(255) values of the gray level values. This process is done on entire pixels in the image. The large matrix refers to image and values enclosed inside a rectangle is considered to be the current processing window. The element encircled refers to processed pixel. Step 2 is illustrated in case (a). Step 3 and 4 are visualized along with the case 1 ) in case (b) .Case II is briefed in case (c.1) and (c.2).

\begin{tabular}{|l|l|l|}
\hline 127 & 255 & 56 \\
\hline 85 & $\mathbf{1 0 6}$ & 75 \\
\hline 23 & 77 & 255 \\
\hline
\end{tabular}

Processing window

\begin{tabular}{|l|l|l|}
\hline 127 & 255 & 56 \\
\hline 85 & $\mathbf{1 0 6}$ & 75 \\
\hline 23 & 77 & 255 \\
\hline
\end{tabular}

output window
Case (a): In the above illustration the processed pixel is checked for $0<$ pxy< 255 . Here in the discussed example processed pixel is 106 . Hence processed pixel is not 0 or 255 . So pixel is considered as noise free and pixel is unaltered.

\begin{tabular}{|l|l|l|}
\hline 177 & 205 & 155 \\
\hline 0 & $\mathbf{2 5 5}$ & 25 \\
\hline 0 & 187 & 124 \\
\hline
\end{tabular}

Processing window

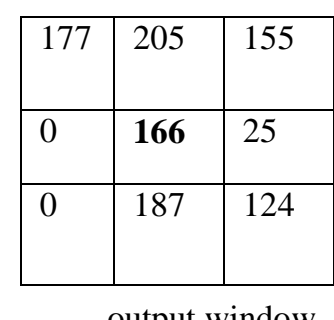

output window
Case (b): In the selected window the processed pixel holds 255 (or 0). So the processed pixel is considered as noisy. Initialize corrupted counter. Convert the $2 \mathrm{D}$ array into $1 \mathrm{D}$ array and sort the converted array. Check for number of noisy pixel inside the window. Every time a 0 or 255 is encountered inside the window the counter value increments by 1 .

Unsorted array: 1770020525518715525124

Sorted array Sxy 0025124155177187205255

Here the count value is 3 so the uncorrupted pixel count (9-3) is 6 then the median value of these uncorrupted pixel is replaced in place of central pixel as output. The case is illustrated as follows. Now check for the presence of 0 or 255 in the sorted array. Every time a $0 \& 255$ is detected the counter is incremented by $1 \mathrm{In}$ the above example there is two 0 and one 255. So counter is holding 3. Now the corrupted pixel is replaced with median of the rank ordered uncorrupted 
pixel in output i.e. corrupted pixel is replaced by median $(25,124,155,177,187,205)=166$

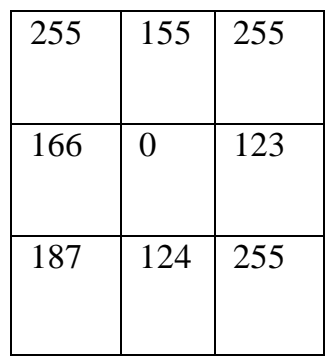

Processing Window

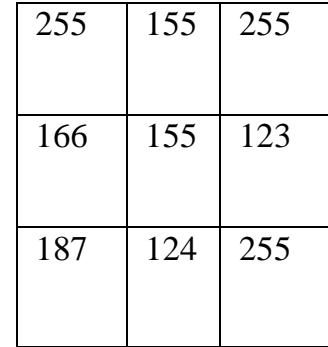

output window
Case (c.1): This case works if the entire pixel inside the current window is either pepper (0) or salt (255). Initialize counter and convert the elements of $2 \mathrm{D}$ window into $1 \mathrm{D}$. Sort the $1 \mathrm{D}$ array. Check for count values by comparing all the pixel elements with 0 or 255 .

Unsorted 1D array: 2551661871550124255123255

Check for Count $=4$ (so case 2 is used )

Sorted 1D array Sxy : 0123124155166187255255255

Uncorrupted pixels : 123124155166187

Now the uncorrupted pixel $\operatorname{count}(9-4)$ is 5. Hence the uncorrupted pixel count is of odd number the noisy noisy centre pixel is replaced with centre of uncorrupted pixels i.e) 155 .

\begin{tabular}{|l|l|l|}
\hline 255 & 255 & 255 \\
\hline 255 & $\mathbf{2 5 5}$ & 255 \\
\hline 255 & 255 & 255 \\
\hline
\end{tabular}

\begin{tabular}{|l|l|l|}
\hline 0 & 0 & 0 \\
\hline 0 & $\mathbf{0}$ & 0 \\
\hline 0 & 0 & 0 \\
\hline
\end{tabular}

Case (c2): if the current processing window has all the pixels to be 0 or 255 then the processing pixel is considered to be texture and left unaltered.

\section{SIMULATION RESULTS}

The Quantitative performance of the proposed algorithm is evaluated based on Peak signal to noise ratio (PSNR) and Mean Square Error (MSE) which is given in equations 1,2 respectively.

$$
\begin{aligned}
& \text { PSNR }=10 \log _{10}\left(\frac{255^{2}}{M S E}\right) \\
& \mathrm{MSE}=\frac{\sum_{i} \sum_{j}\left(r_{i j}-x_{i j}\right) 2}{M \times N}
\end{aligned}
$$

Where $r$ refers to Original image, $\mathrm{n}$ gives the corrupted image $x$ is denotes restored image, $\mathrm{M} \mathrm{x} \mathrm{N}$ is the size of Processed image. The existing algorithms used for the comparison are SMF, AMF, PSMF. The qualitative performance of the proposed algorithm is tested on various images such as Lena, Cameraman, Baboon, Barbara, animal, pepper etc (Images are chosen as per the details of the image). Quantitative analysis is made by varying noise densities in steps of ten from $10 \%$ to $90 \%$ on low detail, given in Table. All the simulation is done in dual CPU E2140@1.6Ghz with 1GB RAM capacity. It is vivid from the tables and graphs that the proposed algorithm suppresses noise at low and high noise densities. Hence the UPCDBF has high PSNR when compared to other algorithms. The qualitative aspect of the UPCDBF against various algorithms for noise densities (10\% to 90\%) for Animal at $90 \%$ is shown in figure.

Table -1: Performance (PSNR) of various algorithms on animal image corrupted

\begin{tabular}{|l|l|l|l|l|l|}
\hline $\begin{array}{l}\text { Noise } \\
\text { Densit } \\
\text { y }\end{array}$ & SMF & AMF & $\begin{array}{l}\text { MEAND } \\
\text { ET }\end{array}$ & $\begin{array}{l}\text { PSM } \\
\text { F }\end{array}$ & PA \\
\hline $10 \%$ & 14.913 & 14.74 & & 18.07 & 33.23 \\
0 & 07 & 17.9894 & 94 & 91 \\
\hline & 11.925 & 11.90 & & 14.91 & 26.85 \\
$20 \%$ & 5 & 73 & 14.8822 & 96 & 66 \\
\hline & 10.183 & 10.15 & & 12.83 & 22.46 \\
$30 \%$ & 5 & 31 & 12.8284 & 86 & 62 \\
\hline & & 8.909 & & 11.18 & 18.97 \\
$40 \%$ & 8.9286 & 1 & 11.1675 & 07 & 25 \\
\hline & & 7.914 & & & 16.21 \\
$50 \%$ & 7.9428 & 3 & 9.7114 & 9.726 & 01 \\
\hline & & & & & 13.79 \\
$60 \%$ & 7.116 & 7.014 & 8.4709 & 8.448 & 23 \\
\hline & & 6.100 & & 7.298 & 11.71 \\
$70 \%$ & 6.3761 & 9 & 7.3373 & 5 & 78 \\
\hline & & 5.070 & & 6.293 & 9.911 \\
$80 \%$ & 5.686 & 6 & 6.3127 & 5 & 3 \\
\hline & & 4.008 & & 5.357 & 8.331 \\
$90 \%$ & 5.0196 & 9 & 5.3833 & 4 & 7 \\
\hline
\end{tabular}



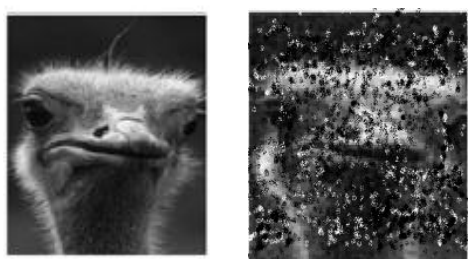

Noise image $\quad 90 \%$ corrupted

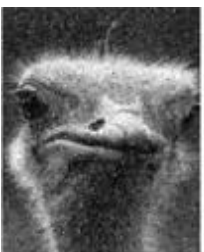

SMF

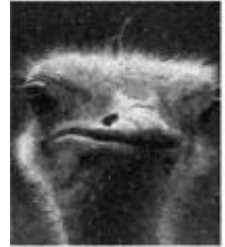

AMF

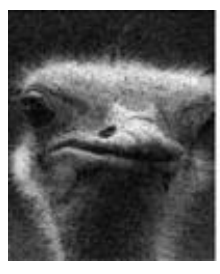

MEANDET

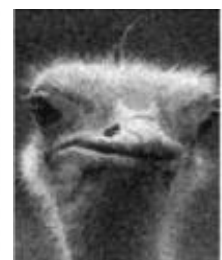

PSMF

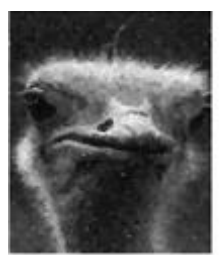

PA

Fig-1 Performance of various filter for animal image corrupted by $90 \%$ Salt and pepper noise

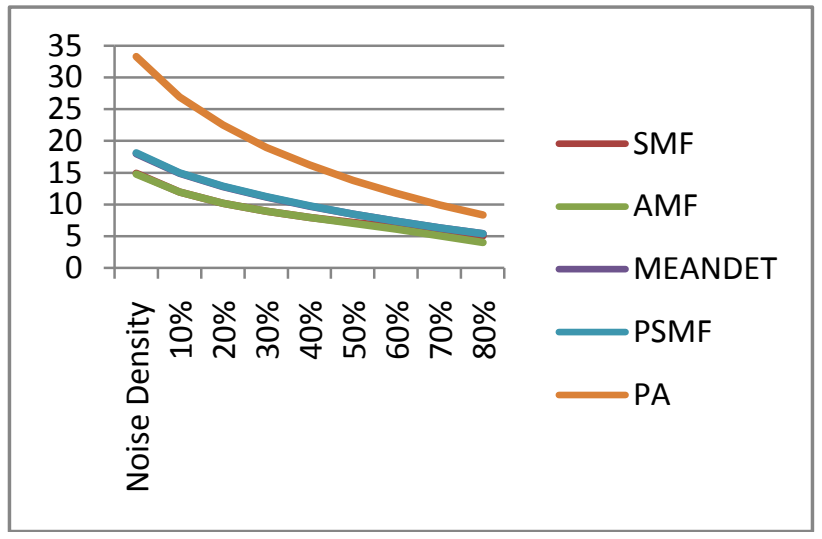

Chart .1 Performance comparison of PSNR value of different filters

\section{CONCLUSIONS}

Uncorrupted pixel count decision based algorithm (UPCDB) using a Mesh based snake like modified shear sorting, having a fixed $3 \times 3$ window is proposed which gives excellent noise suppression capabilities by preserving edges in images corrupted by salt and pepper noise as high as $90 \%$. The Proposed algorithm uses $3 \times 3$ fixed window for increasing noise densities. The proposed algorithm outclasses other existing filters when compared to existing filters both in quantitative and qualitative aspects. The proposed algorithm would also require minimal hardware for implementation

\section{REFERENCES}

[1]. S. J. Astola and P. Kuosmaneen, Fundamentals of non linear Digital Filtering. Boca Raton, FL: CRC, 1997.

[2]. H. Hwang and R. A.Hadded, "Adaptive median filter: New algorithms and results," IEEE transaction on image processing., vol 4,no 4,pp 499-502

[3]. P. E. Ng and K. K. Ma, "A switching median filter with boundary discriminative noise detection for extremely corrupted images," IEEE Transactions on image processing, vol.15, no.6, pp. 1506-1516,June 2006.
[4]. S. Zhang and M.A. Karim, "A new impulse detector for switching median filters," IEEE Signal processing letters, vol.9, no.11, pp. 360-363, November 2002.

[5]. K.S. Srinivasan and D.Ebenezer, "A new fast and efficient decision based algorithm for the removal of high density impulse noise," IEEE Signal processing letters, vol.14, no.3, pp.189-192, March 2007.

[6]. Madhu S., Nair, K.Revathy, Rao. Tatavarti., (2008), “An Improved Decision Based Algorithm for impulse noise removal", Proceedings in Congress on Image and Signal Processing, Pages 426-431, 2008.

[7]. J. Astola and P. Kuosmaneen, Fundamentals of non linear Digital Filtering. Boca Raton, FL: CRC, 1997.

[8] H. Hwang and R. A.Hadded, "Adaptive median filter: New algorithms and results," IEEE transaction on image processing., vol 4,no 4,pp 499-502

[9]. P. E. Ng and K. K. Ma, "A switching median filter with boundary discriminative noise detection for extremely corrupted images," IEEE Transactions on image processing, vol.15, no.6, pp. 1506-1516,June 2006.

[10]. S. Zhang and M.A. Karim, "A new impulse detector for switching median filters," IEEE Signal processing letters, vol.9, no.11, pp. 360-363, November 2002.

[11]. K.S. Srinivasan and D.Ebenezer, "A new fast and efficient decision based algorithm for the removal of high density impulse noise," IEEE Signal processing letters, vol.14, no.3, pp.189-192, March 2007.

[12]. S.Balasubramanian, S.Kalishwaran, R.Muthuraj, D.Ebenezer, V.Jayaraj "An efficient Non linear cascade filtering algorithm for removal of high density salt and pepper noise in image and video sequence", Intl Conf on control, Automation, communication and Energy Conservation,2009, [13]. K.Aiswarya, V .Jayaraj, and D.Ebenezer, "A new and efficient algorithm for the removal of high density salt and pepper noise in images and videos," in second international conference on computer modeling and simulation, 2010, pp.409-413.

[14]. S. Esakkirajan, T. Veerakumar, Adabala N. Subramanyam and C.H. Prem Chand, "Removal of high density Salt and pepper noise through modified decision based Unsymmetrical trimmed median filter." IEEE Signal processing letters, Vol. 18,no.5, May 2011. 
[15]. K.Vasanth, S.Karthik, "Unsymmetrical Trimmed median as Detectors for salt and pepper noise Removal", National Conference on signal and image processing- NCSIP2012, Gandhi gram rural university, February 2012, pages 31-35

[16]. K. K.Vasanth, S.Karthik, SinduDivakaran "Removal of Salt \& Pepper Noise Using Unsymmetrical Trimmed Variants as Detector", European journal of Scientific Research, Vol.70 No.3 (2012), pp. 468-478.

\section{BIOGRAPHIES}

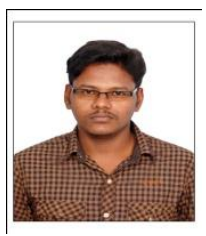

Ramkumar Ramachandran received his B.E. degree in Electronics and communication Engineering from Mailam Engineering College, Anna University, Chennai in 2011 and currently doing his Master degree in Electronics and communication engineering at Manakula vinayagar Institute of technology, Pondicherry University, Puducherry. His field of interest are Image Processing, Wireless and Telecommunication and Computer Networks.

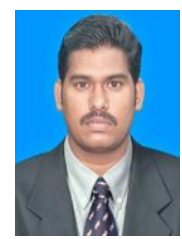

Rajesh Veerappan received his Btech degree in Electronics \& Electrical engineering from Manakula vinayagar engineering college, pondicherry university, Pondicherry in 2007 then received M.E degree in applied electronics from sathyabama university, Chennai in 2012 and currently working as a Assistant Professor at Manakula vinayagar Institute of technology, Pondicherry University, Puducherry. He has four years long experience in the field of teaching. His area of interest includes Image Processing, Electronic circuits, VLSI Design and Embedded core design .

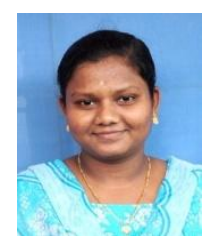

Sharmila Govindaraj received her B.E. degree in Electronics and communication Engineering from Mailam Engineering College, Anna University, Chennai in 2011 and currently doing her Master degree in Electronics and communication engineering at Manakula vinayagar Institute of technology, Pondicherry University, Puducherry. Her field of interest are Image Processing, Digital Signal Processing \& Electronic devices. 